\title{
How to Improve Cord Blood Engraftment?
}

\author{
Meral Beksac ${ }^{1 *}$ and Pinar Yurdakul ${ }^{2 *}$ \\ ${ }^{1}$ Department of Hematology, Ankara University School of Medicine, Ankara, Turkey, ${ }^{2}$ Cord Blood Bank, Ankara University \\ School of Medicine, Ankara, Turkey
}

Various factors make cord blood (CB) a significant source of hematopoietic stem cells (HSCs), including ease of procurement and lack of donor attrition, with the ability to process and store the donor cells long term. Importantly, high proliferative potential of the immature HSCs allows one log less use of cells compared to bone marrow or peripheral blood stem cells. As total nucleated cell (TNC) and $\mathrm{CD}_{3} 4^{+}$cell content of CB grafts are correlated to engraftment rate and speed, strategies to expand HSC and homing have been developed. This chapter will focus only on modalities such as intrabone administration, fucosylation, CD26 inhibition, prostaglandin E2 derivative or

\section{OPEN ACCESS}

Edited by:

Alvin H. Schmaier,

Case Western Reserve University and University Hospitals Case Medical Center, USA

Reviewed by: Owen McCarty, Oregon Health \& Science University,

Mary Joan Laughlin, Cleveland Cord Blood Center, USA

*Correspondence: Meral Beksac meral.beksac@medicine.ankara.edu.tr; Pinar Yurdakul pinar.yurdakul@ankara.edu.tr

Specialty section: This article was submitted to Hematology,

a section of the journal Frontiers in Medicine

Received: 31 October 2015 Accepted: 05 February 2016 Published: 17 February 2016

Citation:

Beksac M and Yurdakul P (2016) How to Improve Cord Blood Engraftment?

Front. Med. 3:7.

doi: 10.3389/fmed.2016.00007 complement 3 exposure, and SDF-1/CXCR4/CXCL-12 pathway interventions that have been experimented successfully. Furthermore, increasing evidence in line with better recognition of CB progenitors that are involved in engraftment and homing will also be addressed.

Keywords: cord blood, engraftment, cord blood transplantation, homing

The first decade of unrelated donor CBT (UCBT) experience was important in defining critical total nucleated cell (TNC) and $\mathrm{CD} 34^{+}$cell dose thresholds required for acceptable clinical outcomes, and in moving from related to UCBT and from pediatric to adult patients. The limitations of this approach also were defined during this period, with low cell dose identified as the critical barrier. Using double CB units (dUCBT) was a solution attempted by a group in Minnesota in 2000. Since then, the method has been proven to be safe and feasible. The advent of dUCBT brought a significant reduction in the risk of graft failure, opening up the possibility of HSCT with CB for essentially all patients without a suitable donor. Nonetheless, the use of dUCBT did not produce faster neutrophil recovery or immune reconstitution. Due to the high cost, it was limited to adult patients to reach the lower threshold. Thus, the advent of dUCBT has led to increased activity in the area of CB graft engineering, especially in the field of ex vivo expansion. Intrinsic and extrinsic cellular factors have been proven to act roles in hematopoietic stem cell (HSC) expansion, thus justifying their role in in vitro and ex vivo culture conditions. Attempts to regulate these factors through ex vivo expansion methods aim to overcome insufficient cell numbers. Delaney et al. achieved significant success justifying the role of triggering Notch-mediated signaling by Notch ligands $(1,2)$. Enhanced generation of CB hematopoietic stem and progenitor cells by culture with StemRegenin 1 and Delta 1 (Ext-IgG) was another ex vivo expansion approach from the same group (3). StemRegenin (HSC835) approach has later been undertaken by the Minnesota group and two Phase I/II clinical trials are ongoing (Clinical Trial Identifier: NCT01930162 and NCT01474681, respectively). In addition, Phase I/II clinical trials to evaluate the impact of infusion of expanded cryopreserved cord blood (CB) progenitor cells with Notch ligand or nicotinamide (NiCord) (Clinical Trial Identifier: NCT 01175785 and NCT01816230, respectively) on augmenting the UCBT outcome are ongoing in Fred Hutchinson and Duke Universities. 
Apart from the expansion protocols applied for increasing cell dose, several other promising notions have also been introduced: optimal selection of HLA matching CB units, killer immunoglobulin-like receptor (KIR) typing of the candidate units; modification of conditioning as well as GVHD prophylaxis regimens; post-transplant use of growth factors/cytokines; and infusion of the CB with accessory mesenchymal stem cells (MSC) are of the most forthcoming modalities as nicely detailed in the recent review by Rocha et al. (4). Results of a clinical trial led by MD Anderson Cancer Center (Clinical Trial Identifier: NCT 00498316) for testing CB expansion on MSC has been addressed in this issue of the journal (5). Despite the fact that many different strategies have gained attention with various fold increases in $\mathrm{CD} 4^{+}$cell numbers, delayed immune reconstitution remains a major challenge after UCBT. There are currently many ongoing in vitro/in vivo experiments along with preclinical and clinical trials for the evaluation of different strategies for improving engraftment after UCBT (6-9). This review aims to summarize the most prominent approaches those having impact on HSC engraftment with the exception of double UCBTs as this approach has become an established transplant modality for patients who lack an HLA matched adult donor.

\section{PREDICTORS OF ENGRAFTMENT}

Generally, TNC and CD34+ are considered to be the best predictors of engraftment standard for selection of units. As discussed in detail in the review by Beksac and Preffer, quantification of HPSC capacity of a graft is still not standardized and differs according to the source of stem cells (10). In the CB, TNC contains a considerable number of normoblasts and lymphocytes. The marrow TNC is more heterogeneous. In $\mathrm{CB}$, the $\mathrm{CD} 34^{+}$per microliter is almost as high as the counts following 3-4 days of G-CSF administration. Migliaccio et al. (11) have compared TNC with colony-forming unit (CFU) numbers and have shown TNC to be inferior to CFU in determining neutrophil and platelet recovery speed following UCBT. Recently, Simmons et al. have identified a CD34 ${ }^{+}$cell subpopulation that co-expresses an antigen (-MA6) (12). This antigen expression predicted platelet engraftment better than $\mathrm{CD} 34^{+}$cell counts. In this study comparison of PBSC with $\mathrm{CB}$, it revealed less frequent MA6 expressing cells within $\mathrm{CB}(<0.2 \%)$ than PBSC ( $8 \%) \mathrm{CD}_{3} 4^{+}$cells. This finding provides an explanation to slower platelet recovery following single UCBTs. The impact of megakaryocytic lineage commitment within $\mathrm{CD}_{3} 4^{+}$ cells following in vitro manipulation of CB is not known yet.

\section{INTRABONE INFUSION}

Historically, the earliest HSCT experience was performed using bone marrow (BM) with direct intrabone infusion. As this approach required technical expertise and induced pain in the recipient, it was later replaced by central venous administration through catheters. Frassoni et al. were the pioneers to revisit intrabone application in UCBT (13). Their preliminary results on 44 patients were able to show neutrophil and platelet recovery times to be 23 and 36 days, respectively. Full donor type chimerism was obtained among all patients. This success tempted investigators to perform a retrospective registry-based analysis, to compare outcomes of results from intrabone single IB-UCBT (n:87) vs. dUCBT (n:149) after myeloablative conditioning regimen (4). Although the median number of TNC were lower among the IB-UCBT $(2.5 \times 10$ vs. $3.9 \times 10 / \mathrm{kg}, P<0.001)$, neutrophil recovery (76 vs. $62 \%, P=0.014$ ), median time to engraftment (23 and 28 days, $P=0.001$ ), and platelet recovery (74 vs. $64 \%$, $P=0.003)$ were better. In multivariate analysis, IB-UCBT was associated with higher neutrophil and platelet recovery and lower acute graft-versus-host disease (II-IV) $(P<0.01)$. IB-UCBT did not increase TRM and there was a trend for longer PFS.

\section{ENHANCING BONE MARROW HOMING}

Full recovery of tri-lineage hematopoiesis following stem cell transplantation depends on proper engraftment of transplanted cells, which relies on engraftment of the most primitive long-term repopulating HSCs (1) To overcome the negating effects of low counts of HSC within CBUs, strategies to improve homing of HSCs to the marrow have been developed. Recently, two new strategies have been proposed. The first is based on inhibition of human analog of the murine-identified HSC-expressed dipeptidylpeptidase (CD26). The second, developed by Minnesota group, is based on ex vivo priming of HSCs before transplantation with small molecules, such as C3 complement fragments, fibrinogen, fibronectin, and hyaluronic acid. This second strategy has been adopted by other groups, such as the investigators in Brigham's Hospital, who have used a prostaglandin E2 derivative to facilitate homing and induce hematopoietic progenitor proliferation (14).

\section{Effects of CD26 and Modification of CXCR4-SDF-1 Axis}

SDF-1, a BM stromal cytokine, plays a significant role in homing of HSCs in BM niche through binding its receptor CXCR-4. It has been suggested that retention of $\mathrm{CB}$ cells in the BM depends highly on the level and SDF-1 binding capacity of CXCR4 after transplantation and allogeneic engraftment (15). Moreover, an efficient SDF-1 gradient and adequate responsiveness of CXCR-4 receptors to SDF-1 in the BM microenvironment are proven to be one of the main cellular events providing engraftment of HSCs in BM $(16,17)$. Through interaction between cells expressing CXCR4 and inflammatory chemotactic molecules, matrix metalloproteinases (MMPs), and angiopoietic factors (e.g., VEGF) SDF-1/CXCR4 axis plays a crucial role by increasing seeding efficiency and speed $(1,17)$.

The Indianapolis team led by Broxmeyer was the first to discover inhibition or deletion of CD26 on donor cells to enhance short-term homing, long-term engraftment, competitive repopulation, secondary transplantation, and mouse survival in an experimental model. Their pivotal findings suggest that CD26 is a novel target for increasing transplantation efficiency. The peptidase CD26 [DPPIV (dipeptidylpeptidase IV)] removes dipeptides from the amino terminus of proteins. They provided evidence that endogenous CD26 expression on donor cells negatively regulated homing and engraftment. CD26 is a widely expressed membrane-bound ectopeptidase that cleaves CXCL12, thereby depleting its chemokine activity and explains the 
homing/engraftment effects. Based on this fact, inhibition of the enzyme (CD26/DPPIV) cleaving SDF-1/CXCL-12 (CXCR4) into a truncated form has been a promising approach to retain the hindered chemotactic effect.

Many different researchers have also provided in vitro and in vivo data that inhibition of CD26/DPPIV clearly enhanced colony stimulating activity as well as engraftment capacity of CB HSCs $(17,18)$. The clinical efficacy of a DPP-IV inhibitor (Sitagliptin), FDA approved for treatment of type II diabetes mellitus, is under investigation in two clinical Phase II studies. Led by Sherif Farag, authors observed a speed up and enhancement of engraftment following CB transplantation (Clinical Trial Identifiers: NCT 00862719 and NCT 01720264) (19). Among 24 patients following a MAC regimen and oral $600 \mathrm{mg}$ Sitagliptin ( -1 to +2 days) suppressed DPP4 activity by $\sim 70-80 \%$, although the effect was not permanent with a return to baseline activity levels within $16 \mathrm{~h}$ of administration. A key finding was a significant correlation between level of DPP4 suppression and neutrophil engraftment $(P=0.002)$. However, the optimal Sitagliptin dose as well as the duration needs to be further investigated clinically $(9,19)$.

SDF-1 regulates the trafficking of pre-B lymphocytes and $\mathrm{T}$ lymphocytes in addition to CD $34^{+}$HSCs. Thus, modulation of cell trafficking by SDF-1, particularly via adhesion molecules, also happens to effect survival of CB T cells in BM environment. These data justifies CXCR4-SDF-1 axis' role in homing and mediating of allogeneic CB engraftment of cells that need to combat recipient immune-mediated graft rejection (15).

Recently, the Leuven group led by C. Verfaille identified a new inhibitor: tissue factor pathway inhibitor (TFPI) that acts as a biological CD26 inhibitor on human marrow or CB hematopoietic progenitor and stem cells. TFPI exerts activity through Glypican-3, which is co-expressed with CD26. Their experimental results have not entered clinical trials yet.

These results suggest that HSC engraftment is not absolute, as previously thought, and indicate that improvement of BM transplant efficiency may be made possible in the clinic.

\section{C3a-C3aR Axis}

One of the major components of the innate immune system is the complement cascade proteins. Treatment with complement proteins, $\mathrm{C} 3 \mathrm{a}$ in particular, has been shown to enhance ex vivo transmigration of $\mathrm{CD} 34^{+}$HSCs from $\mathrm{CB}$ and $\mathrm{BM}$ via elevating the expression of CXCR-4 and MMP-2/MMP-9. Short-term priming of $\mathrm{CB}$-derived $\mathrm{CD} 34^{+}$HSCs may upregulate levels of homing-related molecules. Their ex vivo trans-migratory and in vivo homing potential may overcome the delayed reconstitution after UCB (1).

Ratajczak and co-workers showed that the complement cascade is activated during growth-factor-induced hematopoietic progenitor and HSCs mobilization and that complement cleavage fragments play a part in the mobilization. It was reported that the $\mathrm{C} 3$ a receptor $(\mathrm{C} 3 \mathrm{aR})$ is expressed on $\mathrm{CB} \mathrm{CD} 34^{+}$cells and that CD $34^{+}$-cell migration toward SDF-1 could be enhanced through C3a in vitro (20). In addition, after pretreatment of CB CD34 ${ }^{+}$ cells with a C3aR antagonist (SB290157) homing after transplantation into NOD/SCID mice was impaired $(21,22)$. In the Phase
I clinical trial led by Brunstein et al., the effect of C3a priming was investigated following one primed and one unmanipulated CB among patients with high-risk hematological malignancies (23). In this double CB platform, median time to engraftment was 6 days, which was not significantly different from the time to engraftment of historical non-myeloablative UCBT control patients. Engraftment was dominated by the C3a-primed CBU among nine of 27 evaluable patients. Engraftment was driven by the $\mathrm{CD}^{+}$-cell content of the $\mathrm{CBU} . \mathrm{CD}^{+}$more than $0.5 \times 10^{7}$ cells/ $\mathrm{kg}$ determined the winner (23).

\section{Modulation with Prostaglandin E2}

The stable prostaglandin E2 derivative 16,16-dimethyl prostaglandin E2 (dmPGE2) has been identified as a critical regulator of HSC homeostasis following screening among many chemical molecules in a zebra fish embryo model (24). A brief incubation with dmPGE2 up-regulates the genes responsible from homing (e.g., CXCR4), proliferation (e.g., CyclinD1), and cell survival (e.g., Survivin) (14). Authors hypothesized that brief ex vivo modulation with dmPGE2 could improve patient outcomes by increasing the "effective dose" of HSCs. In a Phase I trial following a RIC dUCBT, they were able to achieve accelerated and long-term neutrophil engraftment ( 17.5 vs. 21 days, $P=0.045)$. The dominating CBUs were those exposed to dmPGE2 in 10/12 of CBTs. Furthermore, there were no adverse events or safety issues related to ex vivo dmPEG2 exposure. A Phase II clinical trial (NCT01627314) is underway to further investigate the feasibility and effectiveness of this manipulation. These authors have expanded their research to discover dmPEG2 priming to modify Wnt signaling resulting in T cell factor (TCF)-mediated transcription. Wnt signaling upregulated interleukin (IL)-7R and IL-2R $\beta$ expression resulting in enhanced survival mediated by the homeostatic cytokines IL-7 and IL-15 (25). This novel approach induces and maintains naive, memory precursors, and long-lived central memory $\mathrm{CD}^{+}$cells. These immune-mediated effects are very promising as immune reconstitution is delayed following CBT and constitutes one of the major problems.

\section{Fucosylation}

Delayed engraftment was shown to be due, at least in part, to low fucosylation (the addition of a fucose molecule) of cell surface molecules important for homing to the BM microenvironment. Interaction among adhesion molecules within the BM microniche depends on the fucosylation of CB HSC. A simple 30-min ex vivo incubation of $\mathrm{CB}$ hematopoietic progenitor cells with fucosyltransferase-VI and its substrate (guanosine diphosphate fucose) is sufficient to increase fucosylation $(26,27)$. Fucosylation has been shown to improve engraftment among irradiated NOD/ SCID mice (4). Moreover, Robinson et al. demonstrated that only fucosylated $\mathrm{CB}$ CD $34^{+}$were responsible from engraftment among NOD-SCID interleukin-2R $\gamma$ (null) mice (28).

In MD Anderson Cancer Center, two Phase II clinical trials are underway to investigate the utility of $\mathrm{CB} \mathrm{CD}_{3} 4^{+}$cell fucosylation (Clinical Trial Identifiers: NCT 01471067 and NCT 02423915, respectively). Expectations are to test clinical utility of fucosylated T cells with an aim to obtain faster engraftment and prevent GVHD among patients with leukemia-lymphoma. 
Encouraging results supporting ex vivo fucosylation in dUCBT setting of the registered NCT 1471067 trial was recently published by Popat et al. (27). Effects of fucosylation were evaluated among 22 patients enrolled in the trial. The median time to neutrophil engraftment was 17 days (range, 12-34 days) compared to 26 days (range, 11-48 days) observed in a control group of 31 patients who had undergone double unmanipulated UCBT ( $P=0.0023)$, Platelet engraftment was also improved: median was 35 days (range, 18-100 days) compared with 45 days (range, 27-120 days) for controls.

\section{CO-ADMINISTRATION OF CB-DERIVED PROGENITOR OR NATURAL KILLER CELLS}

Another strategy to improve engraftment has been based on coadministration of third party stem cells. Robin et al. have demonstrated human stem and progenitor cells are detectable in placental tissue (29). Celgene Cellular Therapeutics (CCT) has developed a proprietary, sterile closed perfusion process for the collection of human placenta-derived stem cells (HPDSCs) from full term placentas. HPDSC are rich in HSCs and hematopoietic progenitor cells, HSC and progenitor CFU forming capabilities, low in HLA Class I and II expression and enhance in vivo engraftment when combined with UCBT in NOD-SCID animals. Elmacken et al. have initiated a pilot study of adding universal donor (third party) HPDSCs with either single or double UCBT following myeloablative or reduced toxicity conditioning in children and adults with selected malignant and non-malignant diseases in a multicenter consortium (IND\#14949; Clinical Trial Identifier NCT 01586455) (30). In their preliminary ASBMT 2015 abstract, they reported a median time to neutrophil engraftment of 22 days following a mean $( \pm \mathrm{SD})$ cell dose of $\mathrm{CD}^{+} 4^{+}\left(3.9 \pm 1.8 \times 10^{5} / \mathrm{kg}\right)$ and HPDSC CD $34^{+}\left(0.3 \pm 0.15 \times 10^{5} / \mathrm{kg}\right)$ infusion.

The use of $\mathrm{CB}$ originated immune cells is one of the most promising immunotherapeutic strategies to be used after CBT (16, 31-33). Induction or adoptive transfer of CB-derived immune cells, particularly natural killer (NK) cells and regulatory $\mathrm{T}$ cells

\section{REFERENCES}

1. Delaney C, Heimfeld S, Brashem-Stein C, Voorhies H, Manger RL, Bernstein ID. Notch-mediated expansion of human cord blood progenitor cells capable of rapid myeloid reconstitution. Nat Med (2010) 16(2):232-6. doi:10.1038/ nm.2080

2. Csaszar E, Wang W, Usenko T, Qiao W, Delaney C, Berstein ID, et al. Blood stem cell fate regulation by Delta-1-mediated rewiring of IL-6 paracrine signaling. Blood (2014) 123(5):650-8. doi:10.1182/blood-2013-08-520445

3. Dahlberg A, Brashem-Stein C, Delaney C, Bernstein ID. Enhanced generation of cord blood hematopoietic stem and progenitor cells by culture with StemRegenin1 and Delta1(Ext-IgG.). Leukemia (2014) 28(10):2097-101. doi:10.1038/leu.2014.181

4. Rocha V, Labopin M, Ruggeri A, Podestà M, Gallamini A, Bonifazi F, et al. Unrelated cord blood transplantation: outcomes after single-unit intrabone injection compared with double-unit intravenous injection in patients with hematological malignancies. Transplantation (2013) 95(10):1284-91. doi:10.1097/TP.0b013e318288ca4d
( $\mathrm{T}$ reg) with or without cytokines are also effective approaches for gaining better engraftment levels after UCBT. All of these approaches have been denoted as "successful" in preclinical in vitro and animal studies (34-36).

The role of NK cells in engraftment is still controversial. Gertow et al. have suggested that mixed chimerism following double UCBT could possibly be related to NK cell tolerance between the CB units; other reports, however, did not show a correlation between KIR ligand incompatibility and engraftment (37). Nonetheless, previous studies of HSC transplantation in mice demonstrated that IL-2-activated NK cells mediate HSC engraftment and that alloreactive NK cells may facilitate engraftment by killing recipient T cells and APCs. As the reduced function and maturation of NK cells arising in the early post-UCBT period can be restored by cytokines, infusion of ex vivo expanded and activated NK cells could represent a means to enhance early engraftment following UCBT.

\section{CONCLUSION}

Until now, many expansion modalities with the ultimate goal of improving engraftment after UCBT were successful to a certain degree. It is of great interest that current evidence supports priming of $\mathrm{CB}$ cells prior to transplantation modulates/improves engraftment dynamics. In addition to generating increased numbers of progenitor cells with rapid in vivo re-population capacity, enhancing HSC homing capacities will improve the kinetics of hematopoietic recovery with better transplant outcome and hopefully less transplant related mortality rates.

\section{AUTHOR CONTRIBUTIONS}

Both authors contributed to the mini review in close collaboration.

\section{FUNDING}

The preparation and publishing of this manuscript was supported by the research funding provided by Turkish Academy of Sciences.

5. de Lima M, McNiece I, Robinson SN, Munsell M, Eapen M, Horowitz M, et al. Cord blood engraftment with ex vivo mesenchymal-cell coculture. $N$ Engl J Med (2012) 367(24):2305-15. doi:10.1056/NEJMoa1207285

6. Ballen KK, Gluckman E, Broxmeyer HE. Umbilical cord blood transplantation: the first 25 years and beyond. Blood (2013) 122:491-8. doi:10.1182/ blood-2013-02-453175

7. Ilic D, Miere C, Lazic E. Umbilical cord blood stem cells: clinical trials in non-hematological disorders. Br Med Bull (2012) 102:43-57. doi:10.1093/ bmb/lds008

8. Oran B, Shpall E. Umbilical cord blood transplantation: a maturing technology. Hematology Am Soc Hematol Educ Program (2012) 2012:215-22. doi:10.1182/ asheducation-2012.1.215

9. Lund TC, Boitano AE, Delaney CS, Shpall EJ, Wagner JE. Advances in umbilical cord blood manipulation-from niche to bedside. Nat Rev Clin Oncol (2015) 12(3):163-74. doi:10.1038/nrclinonc.2014.215

10. Beksac M, Preffer F. Is it time to revisit our current hematopoietic progenitor cell quantification methods in the clinic? Bone Marrow Transplant (2012) 47(11):1391-6. doi:10.1038/bmt.2011.240 
11. Migliaccio AR, Adamson JW, Stevens CE, Dobrila L, Carrier MC, Rubinstein P. Cell dose and speed of engraftment in placental/umbilical cord blood transplantation: graft progenitor cell content is a better predictor than nucleated cell quantity. Blood (2000) 96(8):2717-22.

12. Simmons PJ, Robinson SN, Munsell MF, Thomas MW, Javni JA, Brouard N, et al. Expression of a surface antigen (MA6) by peripheral blood CD34+ cells is correlated with improved platelet engraftment and may explain delayed platelet engraftment following cord blood transplantation. Stem Cells Dev (2015) 24(9):1066-72. doi:10.1089/scd.2014.0439

13. Frassoni F, Gualandi F, Podestà M, Raiola AM, Ibatici A, Piaggio G, et al. Direct intrabone transplant of unrelated cord-blood cells in acute leukaemia: a phase I/II study. Lancet Oncol (2008) 9(9):831-9. doi:10.1016/ S1470-2045(08)70180-3

14. Cutler C, Multani P, Robbins D, Kim HT, Le T, Hoggatt J, et al. Prostaglandinmodulated umbilical cord blood hematopoietic stem cell transplantation. Blood (2013) 122(17):3074-81. doi:10.1182/blood-2013-05-503177

15. Delaney C, Ratajczak MZ, Laughlin MJ. Strategies to enhance umbilical cord blood stem cell engraftment in adult patients. Expert Rev Hematol (2010) 3(3):273-83. doi:10.1586/ehm.10.24

16. Beksac M, Yurdakul P. Modalities to improve cord blood engraftment. J Stem Cell Res Ther (2014) 4:3. doi:10.4172/2157-7633.1000182

17. Campbell TB, Hangoc G, Liu Y, Pollok K, Broxmeyer HE. Inhibition of CD26 in human cord blood CD34+ cells enhances their engraftment of nonobese diabetic/severe combined immunodeficiency mice. Stem Cells Dev (2007) 16(3):347-54. doi:10.1089/scd.2007.9995

18. Jung Y, Wang J, Schneider A, Sun YX, Koh-Paige AJ, Osman NI, et al. Regulation of SDF-1 (CXCL12) production by osteoblasts; a possible mechanism for stem cell homing. Bone (2006) 38(4):497-508. doi:10.1016/j.bone.2005.10.003

19. Farag SS, Srivastava S, Messina-Graham S, Schwartz J, Robertson MJ, Abonour $\mathrm{R}$, et al. In vivo DPP-4 inhibition to enhance engraftment of single-unit cord blood transplants in adults with hematological malignancies. Stem Cells Dev (2013) 22(7):1007-15. doi:10.1089/scd.2012.0636

20. Ratajczak J, Reca R, Kucia M, Majka M, Allendorf DJ, Baran JT, et al. Mobilization studies in mice deficient in either $\mathrm{C} 3$ or C3a receptor (C3aR) reveal a novel role for complement in retention of hematopoietic stem/ progenitor cells in bone marrow. Blood (2004) 103:2071-8. doi:10.1182/ blood-2003-06-2099

21. Reca R, Mastellos D, Majka M, Marquez L, Ratajczak J, Franchini S, et al. Functional receptor for $\mathrm{C} 3 \mathrm{a}$ anaphylatoxin is expressed by normal hematopoietic stem/progenitor cells, and C3a enhances their homing-related responses to SDF-1. Blood (2003) 101(10):3784-93. doi:10.1182/ blood-2002-10-3233

22. Wysoczynski M, Reca R, Lee H, Wu W, Ratajczak J, Ratajczak MZ. Defective engraftment of $\mathrm{C} 3 \mathrm{aR}-/$ - hematopoietic stem progenitor cells shows a novel role of the C3a-C3aR axis in bone marrow homing. Leukemia (2009) 23:1455-61. doi:10.1038/leu.2009.73

23. Brunstein CG, Mckenna DH, DeFor TE, Sumstad D, Paul P, Weisdorf DJ, et al. Complement fragment 3a priming of umbilical cord blood progenitors: safety profile. Biol Blood Marrow Transplant (2013) 19:1474-9. doi:10.1016/j. bbmt.2013.07.016

24. North TE, Goessling W, Walkley CR, Lengerke C, Kopani KR, Lord AM, et al. Prostaglandin E2 regulates vertebrate haematopoietic stem cell homeostasis. Nature (2007) 447(7147):1007-11. doi:10.1038/nature05883

25. Li L, Kim HT, Nellore A, Patsoukis N, Petkova V, McDonough S, et al. Prostaglandin E2 promotes survival of naive UCB T cells via the Wnt $/ \beta$ catenin pathway and alters immune reconstitution after UCBT. Blood Cancer $J$ (2014) 4:e178. doi:10.1038/bcj.2013.75
26. Beksac M. Is there any reason to prefer cord blood instead of adult donors for hematopoietic stem cell transplants? Front Med (2016) 2:95. doi:10.3389/ fmed.2015.00095

27. Popat U, Mehta RS, Rezvani K, Fox P, Kondo K, Marin D, et al. Enforced fucosylation of cord blood hematopoietic cells accelerates neutrophil and platelet engraftment after transplantation. Blood (2015) 125(19):2885-92. doi:10.1182/blood-2015-01-607366

28. Robinson SN, Simmons PJ, Thoma MW, Brouard N, Javni JA, Trilok S, et al. Ex vivo fucosylation improves human cord blood engraftment in NODSCID IL-2Rgamma(null) mice. Exp Hematol (2012) 40(6):445-56. doi:10.1016/j. exphem.2012.01.015

29. Robin C, Bollerot K, Mendes S, Haak E, Crisan M, Cerisoli F, et al. Human placenta is a potent hematopoietic niche containing hematopoietic stem and progenitor cells throughout development. Cell Stem Cell (2009) 5(4):385-95. doi:10.1016/j.stem.2009.08.020

30. Elmacken M, Pulsipher M, Shi Q, Giller R, Moore TB, Harison L, et al. A pilot trial of unrelated cord blood transplantation (UCBT) and unmatched human placental derived stem cells (HPDSC) in children and young adults with malignant and non-malignant disease. Biol Blood Marrow Transplant (2015) 21(Issue 2):S220-1. doi:10.1016/j.bbmt.2014.11.339 Abstract retrieved from Abstracts in 2015 BMT Tandem Meetings database (Accession No, 21: S220)

31. Nadal E. Immunotherapy post-transplant adoptive cellular immunotherapy to harness post-transplant alloreactivity. Revised ed. In: Apperley J, editor. EBMT Hematopoietic Stem Cell Transplantation Book. Paris: EBMT Press (2008). p. $277-86$.

32. Shpall EJ, Bollard CM, Brunstein C. Novel cord blood transplant therapies. Biol Blood Marrow Transplant (2011) 17(1 Suppl):S39-45. doi:10.1016/j. bbmt.2010.10.004

33. Danby R, Rocha V. Improving engraftment and immune reconstitution in umbilical cord blood transplantation. Front Immunol (2014) 5:68. doi:10.3389/ fimmu. 2014.00068

34. Saliba RM, Rezvani K, Leen A, Jorgensen J, Shah N, Hosing C, et al. General and virus-specific immune cell reconstitution after double cord blood transplantation. Biol Blood Marrow Transplant (2015) 21(7):1284-90. doi:10.1016/j. bbmt.2015.02.017

35. Dong S, Maiella S, Xhaard A, Pang Y, Wenandy L, Larghero J, et al. Multiparameter single-cell profiling of human CD4+FOXP3+ regulatory T-cell populations in homeostatic conditions and during graft-versus-host disease. Blood (2013) 122(10):1802-12. doi:10.1182/blood-2013-02-482539

36. Tolar J, Hippen KL, Blazar BR. Immune regulatory cells in umbilical cordblood: T regulatory cells and mesenchymal stromal cells. Br J Haematol (2009) 147(2):200-6. doi:10.1111/j.1365-2141.2009.07781.x

37. Gertow J, Berglund S, Okas M, Uzunel M, Berg L, Karre $K$, et al. Characterization of long-term mixed donor-donor chimerism after double cord blood transplantation. Clin Exp Immunol (2010) 162:146-55. doi:10.1111/j.1365-2249.2010.04212.x

Conflict of Interest Statement: The authors declare that the research was conducted in the absence of any commercial or financial relationships that could be construed as a potential conflict of interest.

Copyright $\odot 2016$ Beksac and Yurdakul. This is an open-access article distributed under the terms of the Creative Commons Attribution License (CC BY). The use, distribution or reproduction in other forums is permitted, provided the original author(s) or licensor are credited and that the original publication in this journal is cited, in accordance with accepted academic practice. No use, distribution or reproduction is permitted which does not comply with these terms. 